\title{
Ag@ZIF-67 decorated cotton fabric as flexible, stable and sensitive SERS substrate for label-free detection of Phenol-soluble modulin
}

\section{Jiangtao Xu}

The Hong Kong Polytechnic University

\section{Songmin Shang}

The Hong Kong Polytechnic University

\section{Wei Gao}

The Hong Kong Polytechnic University

\section{Ping Zeng}

The Hong Kong Polytechnic University

Shouxiang Kinor Jiang ( $\nabla$ kinor.j@polyu.edu.hk)

Hong Hong Polytechnic University

\section{Research Article}

Keywords: flexible SERS substrate, high sensitivity, Ag@ZIF-67, detection of phenol-soluble modulins, label-free

Posted Date: April 1st, 2021

DOI: https://doi.org/10.21203/rs.3.rs-343162/v1

License: (c) (1) This work is licensed under a Creative Commons Attribution 4.0 International License. Read Full License 


\section{Abstract}

Although strategies of compositing noble materials and Zeolitic imidazolate frameworks (ZIFs) have been used to enhance the performance of ZIF-based surface-enhanced Raman scattering (SERS) substrates, the enhancement process still remains unclear and the sampling process with powder-form substrates is challenging for practical applications. In this study, a flexible SERS substrate with silver (Ag) nanoparticle decorated ZIF-67 as the active materials and cotton fabric as the supporting framework is developed with a facile method in two steps. The proposed flexible SERS substrate not only can reduce difficulties during the sampling process, but also expands future applications for sampling towards irregular target substances. Meanwhile, the enhancement mechanism has been investigated by using Methylene Blue (MB) to probe the molecular interactions. The constructed SERS substrate shows the highest enhancement factor of $6.25 \times 10^{6}$ and an excellent detection sensitivity with a limitation of detection (LoD) of $10^{-14} \mathrm{M} / \mathrm{L}$. Because of its excellent performance in SERS tracing, the proposed SERS substrate shows exceptional ability in detecting and identifying phenol-soluble modulins and is considered to be a label-free approach that requires no adding markers/tags or antibodies. The proposed substrate expands the potential applications of SERS technology for rapid detection of bacterial toxins during clinical diagnoses and treatment.

\section{Introduction}

Surface-enhanced Raman scattering (SERS) is an ultrasensitive vibrational spectroscopic technique that is used to detect probe molecules by localizing molecules of interest on an enhanced surface. This technique has been widely explored and used in numerous applications, such as detecting the $\mathrm{pH}$ of biological fluids (Bi et al., 2018), monitoring pollutants (Castro-Grijalba et al., 2020), ensuring food safety (Danaei et al., 2011), detecting cancer biomarkers (Abbosh et al., 2017), nucleic acids (Jiang et al., 2018) and DNA (Cohen et al., 2017), etc. The so-called enhanced surface area is known as a SERS-active substrate, which is the key component of this detection technique. The substrate mainly includes noble metals (Xiong, Chen, Liou, \& Lin, 2017), semiconductors (Yan et al., 2018), graphene (Ling et al., 2010) Mxenes (Soundiraraju \& George, 2017), or their complexes (López-Lorente et al., 2018).

The properties of SERS-active materials, including their sensitivity, stability, reproducibility and portability, play a crucial role in the level of detection and a wide range of practical applications. Meanwhile, those properties have been enhanced through the advancement of SERS-active materials over years.

In terms of sensitivity, ultrahigh sensitivity which is determined through the limitation of detection (LoD) can be mainly achieved by using noble metal nanoparticles (such as silver (Ag) or gold (Au)) (Zhao et al., 2008). Furthermore, the LoD is determined by the number, shape, size, strength and location of "hot spots" on the substrates (Sharma, Frontiera, Henry, Ringe, \& Van Duyne, 2012). Therefore, numerous experiments have been carried out in the literature, and ultrasensitive detection of certain probe molecules, even a single molecule, has been realized with the use of optimized SERS substrates (dos 
Santos, Temperini, \& Brolo, 2019; Moeinian et al., 2019). However, the relatively high cost and low commercial value have inhibited the practical applications of noble metal SERS-active substrates.

The stability of SERS substrates is usually evaluated by examining their Raman signal stability and chemical stability. The former is mainly determined by the distribution of active materials and whether the probe molecules react with the substrate. The chemical stability is mainly affected by the stability of active materials. Although gold nanoparticles have shown exceptional SERS performance (dos Santos et al., 2019; Moeinian et al., 2019), the high cost of noble metal limits their practical applications. Consequently, semiconductor SERS substrates, such as titanium dioxide $\left(\mathrm{TiO}_{2}\right)$ (Ling et al., 2010), iron III oxide $\left(\mathrm{Fe}_{2} \mathrm{O}_{3}\right)\left(\mathrm{Xu}\right.$, Li, et al., 2019), copper telluride (CuTe) (W. Li et al., 2013) and copper I oxide $\left(\mathrm{Cu}_{2} \mathrm{O}\right)$ (Lin et al., 2018) substrates, have been developed. Interestingly, these partial semiconductors present an outstanding LOD of $10^{-7} \mathrm{M}$, high enhancement factor (EF) of $10^{5}$ (Zheng et al., 2017), and excellent chemical and signal stability. However, the fabrication technique of semiconductor SERS substrates with an excellent performance usually involves extreme processing conditions, such as using the hydrothermal method (Cong et al., 2015) and annealing at high temperatures (Ling et al., 2010).

As for signal reproducibility, it is affected by the uniformity of the particle size and distribution of the particles. A considerable number of experiments have been conducted to control the uniformity of the size of the nanoparticles over the years (Z. Chen, Balankura, Fichthorn, \& Rioux, 2019; Z. Chen, Chang, Balasanthiran, Milner, \& Rioux, 2019). Therefore, signal reproducibility has been improved owing to enhanced particle uniformity. Nevertheless, the real challenge in improving the signal reproducibility is the distribution of the particles because SERS-active particles easily aggregate during synthesis. Besides, it is difficult to collect SERS-active particles absorbed with probe molecules, which greatly limits applications in the real world.

For the applications including the examination of food safety (Ogundare \& van Zyl, 2019), the prediction of disease (Kwon et al., 2019), the detection of the presence of bacteria (Zhang et al., 2018) and the monitor of the environment (Xu, Li, et al., 2019), SERS substrates are required to be easy-to-use, light in weight, small in size and have the ability to efficiently collect the target molecules. Thus, the portability or flexibility of SERS substrates matters. Also, flexible SERS substrates, including paper- based (Yu \& White, 2013), polyester (PET) film- based (F. Chen, Li, Belafhal, Chafiq, \& Sun, 2018), tape- based (Liyanage et al., 2018), silicon chip- based (N. Chen et al., 2017) and swab- based (Gong et al., 2014), have been developed by demands of applications in recent years. Although these approaches may have improved the efficiency and reduced the cost of the fabrication of SERS substrates, more works are needed for the evaluation and development of applications with a wider range of target molecules.

To address the limitations of the previous study of SERS substrates, a novel SERS substrate is proposed in this study, taking the processing conditions, the simplicity of fabrication process as well as the SERS performance into consideration. It is reported that metal-organic-frameworks (MOFs), especially zeolitic imidazolate frameworks (ZIFs), can be used as a type of SERS substrate with a high EF of $10^{6}$ and low LOD of $10^{-8}$ (Sun et al., 2019). In previous studies, MOFs are mainly used as an absorption layer placed 
onto noble metal particles because of the porosity (Hu, Liao, Wang, \& Li, 2014). Furthermore, there are few studies focusing on the use of MOFs as SERS substrates. Therefore, regardless of their performance or applications, further related studies need to be carried out. Correspondingly, we combine noble metal nanoparticles and ZIFs with SERS activity and assemble them onto cotton textile to improve their SERS performance. Cotton is an ideal medium to load SERS active particles, for its excellent mechanical properties, large surface area and high degree of flexibility. To clarify the novel flexible SERS substrate, the SERS performance of the ZIF-67/cotton, Ag/cotton and Ag@ZIF-67/cotton samples is systematically investigated, and the enhancement mechanism is also discussed. The application of the proposed substrate for the detection of phenol-soluble modulins (PSMs) as a label-free approach without adding markers/tags or antibodies will validate and expand the practical applications of SERS technology for clinical diagnoses and treatments.

\section{Experimental \\ 2.1 Materials}

Plain woven cotton fabric $(133 \times 72$ counts per square inch, $40 \mathrm{D} \times 40 \mathrm{D})$ was used as the substrate. Cobalt nitrate hexahydrate $\left(\mathrm{Co}\left(\mathrm{NO}_{3}\right)_{2} \cdot 6 \mathrm{H}_{2} \mathrm{O}\right)$ and 2-methymelodoloze (2-MI) (Adamas Co., Ltd.) were used to prepare the solutions. Methanol (AR grade) (Aladdin Co. Ltd.) were also used in the solutions. MB $\left(\mathrm{C}_{16} \mathrm{H}_{18} \mathrm{~N}_{3} \mathrm{~S} \cdot \mathrm{Cl}\right.$ ) (Shanghai Aladdin biochemical Polytron Technologies Inc.) were used to probe the molecular interactions. PSM a1, a3 and a4 with a concentration of $1 \mathrm{mM} / \mathrm{L}$ were purchased from China Peptides Co., Ltd.

\subsection{Fabrication of ZIF-67 and Ag@ZIF-67 coated cotton fabric}

First, $1.5 \mathrm{mmol}$ of $\mathrm{Co}\left(\mathrm{NO}_{3}\right)_{2} \cdot 6 \mathrm{H}_{2} \mathrm{O}$ and $22.5 \mathrm{mmol}$ of 2-MI were dissolved into $20 \mathrm{ml}$ of methanol, and labelled as Solutions A and B, respectively. The cotton fabric samples $(5 \mathrm{~cm} \times 5 \mathrm{~cm})$ were cleaned in an ultrasonic bath with a solution of acetone and ethanol (1:1) for $30 \mathrm{~min}$ at room temperature. The power level and frequency of the ultrasonic treatment were $200 \mathrm{~W}$ and $70 \mathrm{kHz}$, respectively. Then, the fabric samples were washed with deionized water and dried for $12 \mathrm{~h}$ at $50^{\circ} \mathrm{C}$. The cleaned fabric samples were immersed into Solution B for 5 min. Then, Solution A was added by vigorous stirring to Solution B which contained the cotton fabric samples. The mixed system was stored at $35^{\circ} \mathrm{C}$ for $24 \mathrm{~h}$. The treated fabric samples turned purple and were washed 3 times with ethanol and deionized water, respectively. ZIF-67 coated cotton fabric was produced after the fabric was dried at $50^{\circ} \mathrm{C}$ for $12 \mathrm{~h}$.

Then the ZIF-67 coated cotton fabric was deposited with Ag nanoparticles for $5 \mathrm{~s}, 30 \mathrm{~s}, 60 \mathrm{~s}$ and $90 \mathrm{~s}$ by using magnetron sputtering technology with a RF power of $100 \mathrm{~W}$. For comparation, cotton fabric without ZIF-67 was also deposited with Ag nanoparticles at the same conditions. The final samples coated with Ag@ZIF-67 and Ag were labelled as C-ZIF-67, C-AZ5, C-AZ30, C-AZ60, C-AZ90, C-Ag5, C-Ag30, C-Ag60 and 
C-Ag90, where C stands for cotton fabric, Z stands for ZIF-67, A stands for Ag, and the number stands for the coating time of Ag.

\subsection{Characterization}

The crystal structures of the samples were examined by using an X-ray diffractometer (XRD) (Rigaku Smart Lab). The process was carried out with an X'celerator X-ray detector in the normal mode with a $\mathrm{Cu}$ Ka radiation source $(\lambda=0.154 \mathrm{~nm})$ at an accelerating voltage of $45 \mathrm{kV}$, and emission current of $200 \mathrm{~mA}$. Details of the crystal structure were collected at a $2 \theta$ angle range of $5^{\circ}$ to $90^{\circ}$ with a scanned step size of $0.02 \%$ step and a scanned speed of $5 \%$ min. Scanning electron microscopy (SEM) of the ZIF-67 and Ag decorated ZIF-67 coated cotton fabric samples was conducted by using a TESCAN VEGA3 scanning electron microscope. The Raman spectra were collected on a BAYSPEC 3 in 1 Nomadic Raman microscope equipped with $532 \mathrm{~nm}$ and $785 \mathrm{~nm}$ excitation lasers. X-ray photoelectron spectroscopy (XPS) measurements were carried out on a Kratos XSAM800 spectrometer operated at $1486.60 \mathrm{eV}$.

\subsection{Raman measurement}

The Raman behaviors of the ZIF-67 and Ag decorated ZIF-67 coated cotton fabric samples were measured by using $\mathrm{MB}$ as the target molecules. $\mathrm{A} 10^{-3} \mathrm{M}$ solution of the target molecule was first prepared, which was diluted to an aqueous solution with a concentration that ranged from $10^{-4}$ to $10^{-14}$ M. Before measuring the Raman spectra, the sample substrates with dimensions of $1 \mathrm{~cm} \times 1 \mathrm{~cm}$ were soaked in the aqueous solution of target molecule for $5 \mathrm{~min}$.

The Raman spectra were subsequently examined under the Nomadic ${ }^{\mathrm{TM}}$ Raman 3-in-1 microscope. The laser beam was focused onto a spot with a diameter of $\sim 2.6 \mu \mathrm{m}$ to excite the samples through the OLYMPUS MPlanFLN 10x objective lens. The Raman spectra were collected with an integration time of 3 $\mathrm{s}$, and a laser power of $50 \mathrm{~mW}$ at room temperature. The Raman spectra from different locations were collected for each sample.

\section{Results And Discussion}

\subsection{Fabrication of Ag decorated ZIF-67 coated cotton fabric}

Figure 1 shows the fabrication process of the flexible Ag@ZIF-67 decorated cotton fabric substrate. In this study, ZIF-67 is synthesized in accordance with a typical method (Tan, Bennett, \& Cheetham, 2010). To be emphasized, the pre-cleaned cotton fabric was first soaked in a solution of 2-MI to ensure that the cotton fabric was completely saturated with 2-MI for further growth of the ZIF-67 on the surface of the cotton fibers. Then, Ag nanoparticles were deposited onto the obtained C-ZIF-67. The size of the Ag nanoparticles and spacing between them were adjusted by varying the deposition time as done in previous report (Jiang et al., 2015). For comparison purposes, Ag nanoparticles were also deposited onto a pre-cleaned cotton fabric sample without ZIF-67. 
The optical images of the samples are illustrated in Figure S1. Clearly, the color of the cotton fabric changes from white to purple after decorated with ZIF-67, and silver grey after coated with Ag nanoparticles, while the surface of the ZIF-67 is gradually covered after being coated with the Ag nanoparticles, which indicates that the ZIF-67 and Ag nanoparticles were deposited onto the cotton fabric substrate successfully. The flexibility of this substrate is mainly due to the excellent flexibility of the cotton fabric, which will contribute to increasing applications of SERS in tracing chemicals, microorganisms, and toxins on the surface of the target object.

To further validate the color variations of the cotton fabric, which is attributed to the ZIF- 67 and Ag nanoparticles, SEM images of the untreated, ZIF-67 and Ag@ZIF-67 decorated cotton fabric substrates are provided in Figure S2. As presented in Figures S2(a) and S2(b), the smooth morphology of the cotton fabric with a woven structure can be observed. Moreover, a rough fiber surface with polyhedral particles is found after deposited with ZIF-67. However, the smooth morphology with the polyhedral shaped ZIF-67 particles gradually increase in roughness after coated with Ag nanoparticles as shown in Figures S2(c)S2(h), and the morphology of the ZIF-67 particles coated with Ag nanoparticles at a high magnification is shown in Figs. 2(a)-2(d). The figures show that the ZIF-67 particles are covered with Ag nanoparticles that have different sizes with different spacing between the nanoparticles. This shows that ZIF-67 and Ag@ZIF-67 are well coated onto the surface of the cotton fabric.

Furthermore, the crystallinity of the prepared samples was also investigated by examining the XRD patterns; see Fig. $2(\mathrm{e})$. The peaks found at $14.9^{\circ}, 16.7^{\circ}, 22.8^{\circ}$, and $34.6^{\circ}$ belong to the lattice planes (110), (110), (200) and (004) of cellulose, respectively (French, 2014). Meanwhile, the peak at $7.31^{\circ}$ is attributed to the ZIF-67 (Tan et al., 2010). There should also be other ZIF-67 peaks; however, they might have been obscured by the background peaks of cotton and Ag, thus indicating that the ZIF-67 particles have been successfully synthesized. Besides that, the diffraction peaks of Ag located at $38.1^{\circ}, 44.3^{\circ}$, $77.4^{\circ}$ and $81.5^{\circ}$ are also observed (Xu, Jiang, et al., 2019). These results indicate that ZIF-67 and Ag@ZIF67 are well deposited onto the cotton fabric.

\subsection{Evaluation and investigation of SERS performance}

After the C-Ag@ZIF-67 substrate was successfully constructed, its SERS performance was evaluated. In this study, MB, which is a common probe molecular, was used as the target dye tracer (W. Gao, Xu, Cheng, Qiu, \& Jiang, 2020; Xu et al., 2020). The prepared samples were first immersed into the MB solution (10-3 $\mathrm{M} / \mathrm{L}$ ) for $5 \mathrm{~min}$ to absorb the MB molecules, rinsed with deionized water and then subjected to drying at $50^{\circ} \mathrm{C}$ in an $\mathrm{N}_{2}$ atmosphere.

During the process of detecting the Raman signals, incident laser lights with wavelengths of $532 \mathrm{~nm}$ and $785 \mathrm{~nm}$ were applied to focus on the surface of the prepared substrates that were absorbed with the MB molecules, and the scattered signals from the excitation area were recorded. For comparison, C-ZIF-67, CAZ5, C-AZ30, C-AZ60 and C-AZ90, as well as C-Ag5, C-Ag30, C-Ag60, and C-Ag90 were all measured with the Raman spectrometer in the same condition after absorbing the MB molecules. Figure 3 demonstrates the SERS signals of the MB molecules absorbed on the substrates. It can be observed that all of the SERS 
signals are detected on all the samples under a laser excitation of $532 \mathrm{~nm}$ and $785 \mathrm{~nm}$; however, there are different characteristic peaks at the different laser excitation conditions for the same probe molecules. For signals excited at a laser excitation of $532 \mathrm{~nm}$, a highly intense peak is found at $1624 \mathrm{~cm}^{-1}$, while highly intense peaks of MB are observed at $449 \mathrm{~cm}^{-1}$ (P1), $503 \mathrm{~cm}^{-1}$ (P2), $1399 \mathrm{~cm}^{-1}$ (P3), and 1624 $\mathrm{cm}^{-1}(\mathrm{P} 4)$ at a laser excitation of $785 \mathrm{~nm}$. The results are consistent with other reports ( $\mathrm{Y}$. Gao et al., 2018; Xu, Shang, \& Jiang, 2019).

To rule out if there are any effects from the signals of the substrates, the Raman spectra of the substrates were also investigated, see Figure S3. It can be observed in the figure that there are no similar peaks like the characteristic peaks of $\mathrm{MB}$, which confirms that there are no effects from the background.

Furthermore, Fig. 3 shows the intensity of the peak at $1624 \mathrm{~cm}^{-1}$ with $532 \mathrm{~nm}$ laser excitation and P1-P4 with $785 \mathrm{~nm}$ laser excitation which are used to evaluate the SERS enhancement performance. For ZIF-67 and Ag@ZIF-67 coated cotton substrates, it can be observed that the intensity of the MB signals is obviously enhanced, in which the intensity first increases with sputtering time of Ag, then peaks at CAZ60 (60 s of sputtering) and slightly declines at C-A90 (90 s of sputtering). Nevertheless, it was found that the intensity of the signals continues to increase for samples without the presence of ZIF-67. The results indicated that ZIF-67 has good SERS performance and the deposition of Ag nanoparticles can contribute to increase SERS activity. Meanwhile, the results also showed that the effect of the good SERS performance mainly comes from the presence of ZIF-67.

The EF is an important parameter to assess SERS performance and is derived by Eq. (1) (He, $\mathrm{Hu}$, Yao, Wang, \& Yu, 2009; J. Li, Zhang, Lei, \& Li, 2018):

$\mathrm{EF}=\left(\mathrm{I}_{\text {sers }} / \mathrm{N}_{\text {sers }}\right) /\left(\mathrm{I}_{0} / \mathrm{N}_{0}\right)(1)$

where $I_{\text {sers }}$ denotes the SERS intensity of the MB molecules based on the prepared substrates; $I_{0}$ refers to the intensity of the MB molecules based on the untreated cotton fabric; $\mathrm{N}_{\text {sers }}$ denotes the number of $M B$ molecules absorbed on the surface of the SERS substrates, and $\mathrm{N}_{0}$ represents the number of $M B$ molecules with a certain volume that are laser excited. The details of the calculation process can be found in our previous reports (Xu, Li, et al., 2019; Xu et al., 2020; Xu, Shang, et al., 2019). It is worth noting that the peak located at $1624 \mathrm{~cm}^{-1}$ is detected in both the spectra obtained under laser excitation of different wavelengths, thus, the intensity of this peak is selected to calculate the EFs, which are then plotted and the curves are shown in Fig. 4. As shown in the figure, the highest EF of $6.25 \times 10^{6}$ appears in the C-AZ60 samples with an excitation laser of $785 \mathrm{~nm}$. To compare the different enhancement effects of the samples under different laser excitations, the EFs were calculated and are shown in Fig. 4. The figure shows that the EFs of the different samples with an excitation laser of $532 \mathrm{~nm}$ range from $2.2 \times 10^{6}$ to $4.51 \times 10^{6}$, while those with an excitation of $785 \mathrm{~nm}$ laser vary from $1.06 \times 10^{6}$ to $6.25 \times 10^{6}$. For C-ZIF-67, an excitation laser of $532 \mathrm{~nm}$ offers better enhancement. After the samples were sputtered with $\mathrm{Ag}$ nanoparticles for $5 \mathrm{~s}$, the EFs are almost the same, and those under an excitation laser of $785 \mathrm{~nm}$ exceed those under an excitation laser of $532 \mathrm{~nm}$ with longer sputtering time of $\mathrm{Ag}$. 
To investigate the process that produces these phenomenon, the results are assumed to be the synergistic effect of electromagnetic enhancement (EM) and chemical enhancement (CM) according to our previous work (Xu, Shang, et al., 2019), in which the former is derived from the effect of localized surface plasmon resonances (LSPRs) of the noble metal nanoparticles, while the latter is attributed to the charge transfer between the SERS-active materials and probe molecules (Kaneti et al., 2014; L. Yang et al., 2017). In order to obtain more insight into the enhancement performance of the EM effect, Raman mapping was carried out to identify the "hotspots" that appeared due to the Ag nanoparticles, which is the commonly used explanation of the enhancement effect of noble metal particles (J. Li et al., 2018). Raman mapping is an image from the generated spectrum collection after recording each and every scanned pixel of the selected region. As shown in Fig. 5(a), an area of $50 \mu \mathrm{m} \times 50 \mu \mathrm{m}$ is selected. The 3D (Fig. 5(b)) and 2D (Fig. 5(c)) mapping are obtained with pixels of 100 $\times 100$. The bar color changes from blue to red, where blue means the low intensity and red means the high intensity. The high intensity is attributed to the enhancement effect of the Ag nanoparticles. Therefore, the mapping reflects the distribution of the "hotspots". The "hotspots" should be evenly distributed according to the SEM analysis in that the Ag nanoparticles are well distributed on the surface of cotton fabric. However, the excitation laser is focused on the surface of the sample at an angle, and the surface of the fabric appears to be interlaced. Thus, not all of the areas of the fabric surface can be adequately excited and show high intensity at the same time, and the interlaced fibers can also be observed on the 2D mapping image. To determine how the SERS performance would be affected by the sputtering time of Ag, electromagnetic images were obtained by applying the finite-difference time-domain (FDTD) method to investigate the distribution of the electrical field around the Ag nanoparticles after polarized by the used laser. The details of the calculation and simulation are described in the electronic supporting information.

Figures 5(d) and 5(e) show the electric field distributions around the Ag nanoparticles on the surface of the ZIF-67 particles under a laser excitation of $532 \mathrm{~nm}$ and $785 \mathrm{~nm}$. The color of the inserted bar can be observed to be changed from blue to red, which means that the electric field changes from weak to strong. It can be observed that the electric field between the Ag nanoparticles under a laser excitation of $785 \mathrm{~nm}$ is higher than it was excited by a $532 \mathrm{~nm}$ laser, which indicates that the irradiation of a $785 \mathrm{~nm}$ laser will result in stronger polarization between the Ag nanoparticles. Simulated electric field distributions of the Ag nanoparticles without ZIF- 67 are also provided to show whether the ZIF- 67 has any effect, as shown in Figs. 5(f) and 5(g). It was also found that a $785 \mathrm{~nm}$ laser has better polarization ability; meanwhile, the intensity of the electric field was found to be lower than that of the $\mathrm{Ag}$ nanoparticles distributed on ZIF-67, which shows that ZIF-67 affects the polarization of the electric field.

CE is mainly derived from ZIF-67 in this study; therefore, the highest occupied molecular orbital (HOMO) and lowest unoccupied molecular orbital (LUMO) levels of ZIF-67 are calculated to be $4.31 \mathrm{eV}$ and - 5.91 eV, respectively, according to the valance band XPS (Figure S4). As shown in Fig. 5(h), the charge transition from the LUMO of ZIF-67 $(-5.91 \mathrm{eV})$ to the HOMO of MB $(-4.55 \mathrm{eV}$, which is lower than the $\mathrm{HOMO}$ of ZIF-67) can be easily achieved once ZIF-67 is excited by the used light. As well known, the material can only be excited at an energy level that is equal or higher than its band gap energy. Thus, the band gap energy of ZIF-67 was also calculated (Figure S4), which is $1.6 \mathrm{eV}$. The photon energy of $532 \mathrm{~nm}$ 
is $2.33 \mathrm{eV}$, while that of $785 \mathrm{~nm}$ is $1.58 \mathrm{eV}$. Obviously, a $532 \mathrm{~nm}$ laser beam will easily excite ZIF-67, thus resulting in a charge transition, and realizes the charge transfer between the band edges of ZIF-67 and the LUMO of the probe molecules to cause an enhancement of Raman signals, which is in accordance with the SERS performance results.

Additionally, it was found that the Ag nanoparticles loaded on ZIF-67 show a better SERS performance, which might contribute to both EM and CM effects. To properly illustrate this process, the module of the charge transfer between the Ag nanoparticles and ZIF-67 was established, as shown in Fig. 5(i).

Furthermore, this module was established based on the first-principles theory, in which a charge transfer might occur between the Ag nanoparticles and ZIF-67 due to different work functions. The work function of the Ag nanoparticles and ZIF- 67 was calculated to be $4.26 \mathrm{eV}$ and $6.48 \mathrm{eV}$ versus the normal hydrogen electrode (NHE) (Birke \& Lombardi, 2018). Thus, there tends to be charge transfers between the Ag nanoparticles and ZIF-67, which will equilibrate the Fermi level to reach a balance, when the Ag nanoparticles are in directly contact with ZIF-67. Consequently, polarization and charge transfer will occur with the irradiation of the used laser, thus resulting in the enhancement of the local electromagnetic field at the interface between the Ag nanoparticles and ZIF-67, and the SERS performance will be dramatically improved. A comparison with the experimental results shows that the simulation is in good agreement with the original assumption, which demonstrates that the excellent SERS performance is derived from both electromagnetic and chemical enhancements.

\subsection{SERS sensitivity evaluation of C-Ag@ZIF-67 substrates}

$\mathrm{MB}$ solutions with a concentration that ranged from $10^{-14} \mathrm{M}$ to $10^{-3} \mathrm{M}$ were used to evaluate the sensitivity of the prepared flexible SERS substrates. The obtained spectra are shown in Fig. 6(a), and the spectra of the MB with a concentration that ranges from $10^{-14} \mathrm{M}$ to $10^{-12} \mathrm{M}$ are also provided in Fig. 6(b) for clarity. On the C-Ag@ZIF-67 substrates, MB can be detected even at a low concentration of $10^{-14} \mathrm{M}$, which was not easily done in other research works. Figure $6(\mathrm{c})$ shows a comparison of the LOD and EF results between this study and those of other studies. It can be seen that the performance of the prepared SERS substrate in this study is higher than the others, thus indicating its excellent detection sensitivity (Cai et al., 2018; Guselnikova et al., 2019; D. Li et al., 2019; Q. Li et al., 2019; Z. Yang, Liu, Wang, \& Zhang, 2020).

\subsection{Evaluation of signal reproducibility and stability}

The SERS performance of the C-Ag@ZIF-67 substrates was also examined through their signal reproducibility and stability, which plays an important role in practical applications. The SERS spectra of the prepared substrates absorbed with a $10^{-3} \mathrm{M}$ MB solution were collected by using 30 different locations on the same sample to evaluate the reproducibility, as shown in Fig. 7(a). The variations in the intensity of the peak at $-1624 \mathrm{~cm}^{-1}$ were further established and the relative standard deviation (RSD) was calculated to evaluate the reproducibility. It is observed in Fig. 7(b) that the intensity of each peak fluctuates slightly with RSD values that range from $2.7-3.8 \%$, thus indicating a good reproducibility. 
To evaluate the signal stability of the C-Ag@ZIF-67 substrates, Raman spectra of the MB molecules on the SERS substrate were recorded in the same region with changes in time; that is, for 10 min at intervals of 1 min, as shown in Fig. 7(c). It can be observed that characteristic peaks of MB are the same during the process. The intensity of the characteristic peaks is individually plotted to determine the variation during continuous laser irradiation (Fig. 7(d)). The RSD values of the different peaks are also calculated to assess the stability. The RSD values of P1-P4 are 3.7\%, 5.7\%, 4.2\% and 5.1\%, respectively, which indicate a relatively high stability of the signals.

\subsection{Detection and identification of phenol-soluble modulin peptides}

Staphylococcus aureus (S. aureus), especially superbugs, is a severe threat causing infections. PSM peptides, which are produced by $\mathrm{S}$. aureus, are the main virulence factor that causes inflammation, lysis of human cells by altering the cell cycle and structuring biofilm during the treatment of infections (Cheung, Joo, Chatterjee, \& Otto, 2014; Schwartz, Syed, Stephenson, Rickard, \& Boles, 2012). Its PSM family members, including PSMa1, PSMa3, and PSMa4, are formed from four peptides with about 20residues in length. These PSMs play an important role in contributing to the emergence of methicillin resistant $\mathrm{S}$. aureus, which is responsible for infections that are resistant to treatment, otherwise known as superbugs (Wang et al., 2007). Although many studies have been done to investigate the composition and structure of PSMas, there is still an absence of a rapid detection method that can identify their presence during clinical diagnosis and treatment. Therefore, the current structured SERS substrate in this study is investigated to determine its ability to identify PSMas based on provided information (Salinas, Colletier, Moshe, \& Landau, 2018; Tayeb-Fligelman et al., 2017; Yao et al., 2019). Here, PSMa1, PSMa3, and PSMa4 are selected as the target objects. Solutions made of PSMas with a concentration of $1 \mathrm{mM} / \mathrm{L}$ were prepared, and $10 \mu \mathrm{L}$ of the PSM solution was dripped onto the surface of C-AZ60, followed by testing through Raman spectroscopy.

The structure, peptide sequence and corresponding Raman spectrum of PSM a1, a3 and a4 are shown in Fig. 8. As shown in the figure, the different PSMs have a different permutation structure with similar amino acids. Without referring to the obtained SERS spectra, the PSMs can only be identified using protein sequencing technology to analyze their different amino acid combinations. However, such a sequencing analysis is a complex and time-consuming process which requires the use of special equipment and can only be completed by a professional technician. Therefore, it is not conducive to rapid clinical diagnoses. Here, it can be observed in the SERS spectra that different PSMs have been detected and identified by the different peptide fingerprints as marked in the Figs. 8(a), 8(b) and 8(c) with different color for different PSMs at the same range of Raman shift. For clarify, the spectra ranged from $760 \mathrm{~cm}^{-}$

$1 \sim 1060 \mathrm{~cm}^{-1}$ and $1330 \mathrm{~cm}^{-1} \sim 1540 \mathrm{~cm}^{-1}$ were individually presented in Figs. 8 (d) and (e), respectively. As shown in Fig. 8 (d), the difference of fingerprints of different PSMs can be told that there is a peak located around $829 \mathrm{~cm}^{-1}$ for PSM a4 and two peaks located around $829 \mathrm{~cm}^{-1}$ and $1003 \mathrm{~cm}^{-1}$ for PSM a3, while there is no obvious peaks for PSM a1. Meanwhile, as shown in Fig. 8 (e), PSM a4 shows a peak 
located around $1458 \mathrm{~cm}^{-1}$, PSM a3 shows two peaks located around $1396 \mathrm{~cm}^{-1}$ and $1455 \mathrm{~cm}^{-1}$, while PSM a1 contains four peaks located around $1358 \mathrm{~cm}^{-1}, 1400 \mathrm{~cm}^{-1}, 1460 \mathrm{~cm}^{-1}$ and $1519 \mathrm{~cm}^{-1}$ respectively. However, it is difficult to pinpoint the specific characteristics of the SERS spectra of these peptide fingerprints because the obtained SERS spectra are not only affected by the composition, but also subjected to the 2D or 3D structures of these peptides (Guerrini et al., 2013). Despite this, this study has confirmed that SERS technology can be applied to identify the PSMs of S. aureus by using a labelfree technique that does not require adding markers/tags or antibodies. This study therefore provides important experimental evidence to increase the potential applications of SERS technology, which has never been reported in the literature to date.

\section{Conclusion}

In summary, a flexible SERS substrate, which shows great potential for detecting and identifying PSMs, has been successfully constructed with a simple method within two steps. ZIF-67 was first assembled on the surface of cotton fabric, and Ag nanoparticles were deposited onto the surface of ZIF- 67 by modifying the particle size and gap between the particles by varying the deposition time. Then the SERS performance was evaluated via Raman spectroscopy of the MB molecule. With different sputtering times

to deposit the Ag nanoparticles, we found that the C-ZIF-67 sample sputtered with Ag nanoparticles for 60 $s$ had the best response to the MB molecule (as the probe) during Raman measurements. Besides, the CAZ60 SERS substrate also demonstrated the ability to trace the signals of PSMs, which means that this substrate has excellent potential for detecting and identifying bacterial toxins during clinical diagnoses and treatment.

\section{Declarations}

\section{Acknowledgements}

This work was supported by the Hong Kong Innovation Technology Funding (PRP/104/20TI).

\section{References}

Abbosh, C., Birkbak, N. J., Wilson, G. A., Jamal-Hanjani, M., Constantin, T., Salari, R., ... The, T. c. (2017). Phylogenetic ctDNA analysis depicts early-stage lung cancer evolution. Nature, 545(7655), 446-451. doi:10.1038/nature22364

Bi, L., Wang, Y., Yang, Y., Li, Y., Mo, S., Zheng, Q., \& Chen, L. (2018). Highly Sensitive and Reproducible SERS Sensor for Biological pH Detection Based on a Uniform Gold Nanorod Array Platform. ACS Applied Materials \& Interfaces, 10(18), 15381-15387. doi:10.1021/acsami.7b19347

Birke, R. L., \& Lombardi, J. R. (2018). TDDFT Study of Charge-Transfer Raman Spectra of 4Mercaptopyridine on Various ZnSe Nanoclusters as a Model for the SERS of 4-Mpy on Semiconductors. 
The Journal of Physical Chemistry C, 122(9), 4908-4927. doi:10.1021/acs.jpcc.7b12392

Cai, Y., Wu, Y., Xuan, T., Guo, X., Wen, Y., \& Yang, H. (2018). Core-Shell Au@Metal-Organic Frameworks for Promoting Raman Detection Sensitivity of Methenamine. ACS Applied Materials \& Interfaces, 10(18), 15412-15417. doi:10.1021/acsami.8b01765

Castro-Grijalba, A., Montes-García, V., Cordero-Ferradás, M. J., Coronado, E., Pérez-Juste, J., \& PastorizaSantos, I. (2020). SERS-Based Molecularly Imprinted Plasmonic Sensor for Highly Sensitive PAH Detection. ACS Sensors, 5(3), 693-702. doi:10.1021/acssensors.9b01882

Chen, F., Li, J., Belafhal, A., Chafiq, A., \& Sun, X. (2018). Near-field spectral shift of a zero-order Bessel beam scattered from a spherical particle. Laser Physics Letters, 15(6), 066002. doi:10.1088/1612202x/aaa9d1

Chen, N., Ding, P., Shi, Y., Jin, T., Su, Y., Wang, H., \& He, Y. (2017). Portable and Reliable Surface-Enhanced Raman Scattering Silicon Chip for Signal-On Detection of Trace Trinitrotoluene Explosive in Real Systems. Analytical Chemistry, 89(9), 5072-5078. doi:10.1021/acs.analchem.7b00521

Chen, Z., Balankura, T., Fichthorn, K. A., \& Rioux, R. M. (2019). Revisiting the Polyol Synthesis of Silver Nanostructures: Role of Chloride in Nanocube Formation. ACS Nano, 13(2), 1849-1860. doi:10.1021/acsnano.8b08019

Chen, Z., Chang, J. W., Balasanthiran, C., Milner, S. T., \& Rioux, R. M. (2019). Anisotropic Growth of Silver Nanoparticles Is Kinetically Controlled by Polyvinylpyrrolidone Binding. Journal of the American Chemical Society, 141(10), 4328-4337. doi:10.1021/jacs.8b11295

Cheung, G. Y. C., Joo, H.-S., Chatterjee, S. S., \& Otto, M. (2014). Phenol-soluble modulins - critical determinants of staphylococcal virulence. FEMS Microbiology Reviews, 38(4), 698-719.

doi:10.1111/1574-6976.12057

Cohen, J. D., Javed, A. A., Thoburn, C., Wong, F., Tie, J., Gibbs, P., . . Lennon, A. M. (2017). Combined circulating tumor DNA and protein biomarker-based liquid biopsy for the earlier detection of pancreatic cancers. Proceedings of the National Academy of Sciences, 114(38), 10202-10207. doi:10.1073/pnas.1704961114

Cong, S., Yuan, Y., Chen, Z., Hou, J., Yang, M., Su, Y., . . Zhao, Z. (2015). Noble metal-comparable SERS enhancement from semiconducting metal oxides by making oxygen vacancies. Nature Communications, 6(1), 7800. doi:10.1038/ncomms8800

Danaei, G., Finucane, M. M., Lu, Y., Singh, G. M., Cowan, M. J., Paciorek, C. J., . . Ezzati, M. (2011). National, regional, and global trends in fasting plasma glucose and diabetes prevalence since 1980: systematic analysis of health examination surveys and epidemiological studies with 370 country-years 
and $2 \cdot 7$ million participants. The Lancet, 378(9785), 31-40. doi:https://doi.org/10.1016/S01406736(11)60679-X

dos Santos, D. P., Temperini, M. L. A., \& Brolo, A. G. (2019). Intensity Fluctuations in Single-Molecule Surface-Enhanced Raman Scattering. Accounts of Chemical Research, 52(2), 456-464. doi:10.1021/acs.accounts.8b00563

French, A. D. (2014). Idealized powder diffraction patterns for cellulose polymorphs. Cellulose, 21(2), 885896. doi:10.1007/s10570-013-0030-4

Gao, W., Xu, J., Cheng, C., Qiu, S., \& Jiang, S. (2020). Rapid and highly sensitive SERS detection of fungicide based on flexible "wash free" metallic textile. Applied Surface Science, 512, 144693. doi:https://doi.org/10.1016/j.apsusc.2019.144693

Gao, Y., Gao, N., Li, H., Yuan, X., Wang, Q., Cheng, S., \& Liu, J. (2018). Semiconductor SERS of diamond. Nanoscale, 10(33), 15788-15792. doi:10.1039/C8NR04465A

Gong, Z., Du, H., Cheng, F., Wang, C., Wang, C., \& Fan, M. (2014). Fabrication of SERS Swab for Direct Detection of Trace Explosives in Fingerprints. ACS Applied Materials \& Interfaces, 6(24), 21931-21937. doi:10.1021/am507424v

Guerrini, L., Pazos, E., Penas, C., Vázquez, M. E., Mascareñas, J. L., \& Alvarez-Puebla, R. A. (2013). Highly Sensitive SERS Quantification of the Oncogenic Protein c-Jun in Cellular Extracts. Journal of the American Chemical Society, 135(28), 10314-10317. doi:10.1021/ja405120x

Guselnikova, O., Postnikov, P., Elashnikov, R., Miliutina, E., Svorcik, V., \& Lyutakov, O. (2019). Metal-organic framework (MOF-5) coated SERS active gold gratings: A platform for the selective detection of organic contaminants in soil. Analytica Chimica Acta, 1068, 70-79. doi:https://doi.org/10.1016/j.aca.2019.03.058

He, D., Hu, B., Yao, Q.-F., Wang, K., \& Yu, S.-H. (2009). Large-Scale Synthesis of Flexible Free-Standing SERS Substrates with High Sensitivity: Electrospun PVA Nanofibers Embedded with Controlled Alignment of Silver Nanoparticles. ACS Nano, 3(12), 3993-4002. doi:10.1021/nn900812f

Hu, Y., Liao, J., Wang, D., \& Li, G. (2014). Fabrication of Gold Nanoparticle-Embedded Metal-Organic Framework for Highly Sensitive Surface-Enhanced Raman Scattering Detection. Analytical Chemistry, 86(8), 3955-3963. doi:10.1021/ac5002355

Jiang, S., Miao, D., Yang, G., Chen, Z., Li, A., \& Shang, S. (2015). Fabrication of Ag thin film on polyester fabric by roll to roll magnetron sputtering system. Journal of Materials Science: Materials in Electronics, 26(5), 3364-3369. doi:10.1007/s10854-015-2841-6

Jiang, S., Xu, J., Chen, Z., Guo, R., Miao, D., Peng, L., . . Shang, S. (2018). Enhanced electro-conductivity and multi-shielding performance with copper, stainless steel and titanium coating onto PVA impregnated 
cotton fabric. Journal of Materials Science: Materials in Electronics, 29(7), 5624-5633. doi:10.1007/s10854-018-8531-4

Kaneti, Y. V., Zakaria, Q. M. D., Zhang, Z., Chen, C., Yue, J., Liu, M., . . Yu, A. (2014). Solvothermal synthesis of ZnO-decorated a-Fe2O3 nanorods with highly enhanced gas-sensing performance toward nbutanol. Journal of Materials Chemistry A, 2(33), 13283-13292. doi:10.1039/C4TA01837K

Kwon, G., Kim, J., Kim, D., Ko, Y., Yamauchi, Y., \& You, J. (2019). Nanoporous cellulose paper-based SERS platform for multiplex detection of hazardous pesticides. Cellulose, 26(8), 4935-4944.

doi:10.1007/s10570-019-02427-8

Li, D., Cao, X., Zhang, Q., Ren, X., Jiang, L., Li, D., . . Liu, H. (2019). Facile in situ synthesis of core-shell MOF@Ag nanoparticle composites on screen-printed electrodes for ultrasensitive SERS detection of polycyclic aromatic hydrocarbons. Journal of Materials Chemistry A, 7(23), 14108-14117. doi:10.1039/C9TA03690C

Li, J., Zhang, W., Lei, H., \& Li, B. (2018). Ag nanowire/nanoparticle-decorated MoS2 monolayers for surface-enhanced Raman scattering applications. Nano Research, 11(4), 2181-2189.

doi:10.1007/s12274-017-1836-4

Li, Q., Gong, S., Zhang, H., Huang, F., Zhang, L., \& Li, S. (2019). Tailored necklace-like Ag@ZIF-8 core/shell heterostructure nanowires for high-performance plasmonic SERS detection. Chemical Engineering Journal, 371, 26-33. doi:https://doi.org/10.1016/j.cej.2019.03.236

Li, W., Zamani, R., Rivera Gil, P., Pelaz, B., Ibáñez, M., Cadavid, D., . . Cabot, A. (2013). CuTe Nanocrystals: Shape and Size Control, Plasmonic Properties, and Use as SERS Probes and Photothermal Agents. Journal of the American Chemical Society, 135(19), 7098-7101. doi:10.1021/ja401428e

Lin, J., Hao, W., Shang, Y., Wang, X., Qiu, D., Ma, G., . . Guo, L. (2018). Direct Experimental Observation of Facet-Dependent SERS of Cu2O Polyhedra. Small, 14(8), 1703274. doi:https://doi.org/10.1002/smll.201703274

Ling, X., Xie, L., Fang, Y., Xu, H., Zhang, H., Kong, J., . . Liu, Z. (2010). Can Graphene be used as a Substrate for Raman Enhancement? Nano Letters, 10(2), 553-561. doi:10.1021/nl903414x

Liyanage, T., Rael, A., Shaffer, S., Zaidi, S., Goodpaster, J. V., \& Sardar, R. (2018). Fabrication of a selfassembled and flexible SERS nanosensor for explosive detection at parts-per-quadrillion levels from fingerprints. Analyst, 143(9), 2012-2022. doi:10.1039/C8AN00008E

López-Lorente, A. I., Picca, R. A., Izquierdo, J., Kranz, C., Mizaikoff, B., Di Franco, C., . . Valentini, A. (2018). lon beam sputtering deposition of silver nanoparticles and $\mathrm{TiOx} / \mathrm{ZnO}$ nanocomposites for use in surface enhanced vibrational spectroscopy (SERS and SEIRAS). Microchimica Acta, 185(2), 153.

doi:10.1007/s00604-018-2708-7 
Moeinian, A., Gür, F. N., Gonzalez-Torres, J., Zhou, L., Murugesan, V. D., Dashtestani, A. D., . . Strehle, S. (2019). Highly Localized SERS Measurements Using Single Silicon Nanowires Decorated with DNA Origami-Based SERS Probe. Nano Letters, 19(2), 1061-1066. doi:10.1021/acs.nanolett.8b04355

Ogundare, S. A., \& van Zyl, W. E. (2019). A review of cellulose-based substrates for SERS: fundamentals, design principles, applications. Cellulose, 26(11), 6489-6528. doi:10.1007/s10570-019-02580-0

Salinas, N., Colletier, J.-P., Moshe, A., \& Landau, M. (2018). Extreme amyloid polymorphism in Staphylococcus aureus virulent PSMa peptides. Nature Communications, 9(1), 3512. doi:10.1038/s41467-018-05490-0

Schwartz, K., Syed, A. K., Stephenson, R. E., Rickard, A. H., \& Boles, B. R. (2012). Functional Amyloids Composed of Phenol Soluble Modulins Stabilize Staphylococcus aureus Biofilms. PLOS Pathogens, 8(6), e1002744. doi:10.1371/journal.ppat.1002744

Sharma, B., Frontiera, R. R., Henry, A.-I., Ringe, E., \& Van Duyne, R. P. (2012). SERS: Materials, applications, and the future. Materials Today, 15(1), 16-25. doi:https://doi.org/10.1016/S1369-7021(12)70017-2

Soundiraraju, B., \& George, B. K. (2017). Two-Dimensional Titanium Nitride (Ti2N) MXene: Synthesis, Characterization, and Potential Application as Surface-Enhanced Raman Scattering Substrate. ACS Nano, 11(9), 8892-8900. doi:10.1021/acsnano.7b03129

Sun, H., Cong, S., Zheng, Z., Wang, Z., Chen, Z., \& Zhao, Z. (2019). Metal-Organic Frameworks as Surface Enhanced Raman Scattering Substrates with High Tailorability. Journal of the American Chemical Society, 141(2), 870-878. doi:10.1021/jacs.8b09414

Tan, J. C., Bennett, T. D., \& Cheetham, A. K. (2010). Chemical structure, network topology, and porosity effects on the mechanical properties of Zeolitic Imidazolate Frameworks. Proceedings of the National Academy of Sciences, 107(22), 9938-9943. doi:10.1073/pnas.1003205107

Tayeb-Fligelman, E., Tabachnikov, O., Moshe, A., Goldshmidt-Tran, O., Sawaya, M. R., Coquelle, N., .. . Landau, M. (2017). The cytotoxic <em>Staphylococcus aureus</em> PSMa3 reveals a cross-a amyloidlike fibril. Science, 355(6327), 831-833. doi:10.1126/science.aaf4901

Wang, R., Braughton, K. R., Kretschmer, D., Bach, T.-H. L., Queck, S. Y., Li, M., . . Otto, M. (2007). Identification of novel cytolytic peptides as key virulence determinants for community-associated MRSA. Nature Medicine, 13(12), 1510-1514. doi:10.1038/nm1656

Xiong, Z., Chen, X., Liou, P., \& Lin, M. (2017). Development of nanofibrillated cellulose coated with gold nanoparticles for measurement of melamine by SERS. Cellulose, 24(7), 2801-2811. doi:10.1007/s10570017-1297-7

Xu, J., Jiang, S., Peng, L., Wang, Y., Shang, S., Miao, D., \& Guo, R. (2019). AgNps-PVA-coated woven cotton fabric: Preparation, water repellency, shielding properties and antibacterial activity. Journal of 
Industrial Textiles, 48(10), 1545-1565. doi:10.1177/1528083718764908

Xu, J., Li, X., Wang, Y., Guo, R., Shang, S., \& Jiang, S. (2019). Flexible and reusable cap-like thin Fe2O3 film for SERS applications. Nano Research, 12(2), 381-388. doi:10.1007/s12274-018-2227-1

Xu, J., Li, X., Wang, Y., Hua, T., Guo, R., Miao, D., \& Jiang, S. (2020). Flexible, stable and sensitive surfaceenhanced Raman scattering of graphite/titanium-cotton substrate for conformal rapid food safety detection. Cellulose, 27(2), 941-954. doi:10.1007/s10570-019-02836-9

Xu, J., Shang, S., \& Jiang, S. (2019). A stable, ultrasensitive and flexible substrate integrated from 1D Ag/ a-Fe2O3/SiO2 fibers for practical surface-enhanced Raman scattering detection. Composites Part B: Engineering, 177, 107376. doi:https://doi.org/10.1016/j.compositesb.2019.107376

Yan, X., Xu, Y., Tian, B., Lei, J., Zhang, J., \& Wang, L. (2018). Operando SERS self-monitoring photocatalytic oxidation of aminophenol on TiO2 semiconductor. Applied Catalysis B: Environmental, 224, 305-309. doi:https://doi.org/10.1016/j.apcatb.2017.10.009

Yang, L., Wang, W., Jiang, H., Zhang, Q., Shan, H., Zhang, M., . . Sun, Z. (2017). Improved SERS performance of single-crystalline TiO2 nanosheet arrays with coexposed $\{001\}$ and $\{101\}$ facets decorated with Ag nanoparticles. Sensors and Actuators B: Chemical, 242, 932-939. doi:https://doi.org/10.1016/j.snb.2016.09.162

Yang, Z., Liu, T., Wang, W., \& Zhang, L. (2020). Stacked hexagonal prism of Ag@Ni-MOF-1 as functionalized SERS platform through rational integration of catalytic synthesis of dopamine-quinone at physiological pH with a biomimetic route. Chemical Communications, 56(20), 3065-3068. doi:10.1039/C9CC09145A

Yao, Z., Cary, B. P., Bingman, C. A., Wang, C., Kreitler, D. F., Satyshur, K. A., . . Gellman, S. H. (2019). Use of a Stereochemical Strategy To Probe the Mechanism of Phenol-Soluble Modulin a3 Toxicity. Journal of the American Chemical Society, 141(19), 7660-7664. doi:10.1021/jacs.9b00349

Yu, W. W., \& White, I. M. (2013). Inkjet-printed paper-based SERS dipsticks and swabs for trace chemical detection. Analyst, 138(4), 1020-1025. doi:10.1039/C2AN36116G

Zhang, Z., Han, X., Wang, Z., Yang, Z., Zhang, W., Li, J., . . Xing, B. (2018). A live bacteria SERS platform for the in situ monitoring of nitric oxide release from a single MRSA. Chemical Communications, 54(51), 7022-7025. doi:10.1039/C8CC02855A

Zhao, J., Pinchuk, A. O., McMahon, J. M., Li, S., Ausman, L. K., Atkinson, A. L., \& Schatz, G. C. (2008). Methods for Describing the Electromagnetic Properties of Silver and Gold Nanoparticles. Accounts of Chemical Research, 41(12), 1710-1720. doi:10.1021/ar800028j

Zheng, Z., Cong, S., Gong, W., Xuan, J., Li, G., Lu, W., . . Zhao, Z. (2017). Semiconductor SERS enhancement enabled by oxygen incorporation. Nature Communications, 8(1), 1993. 
Figures

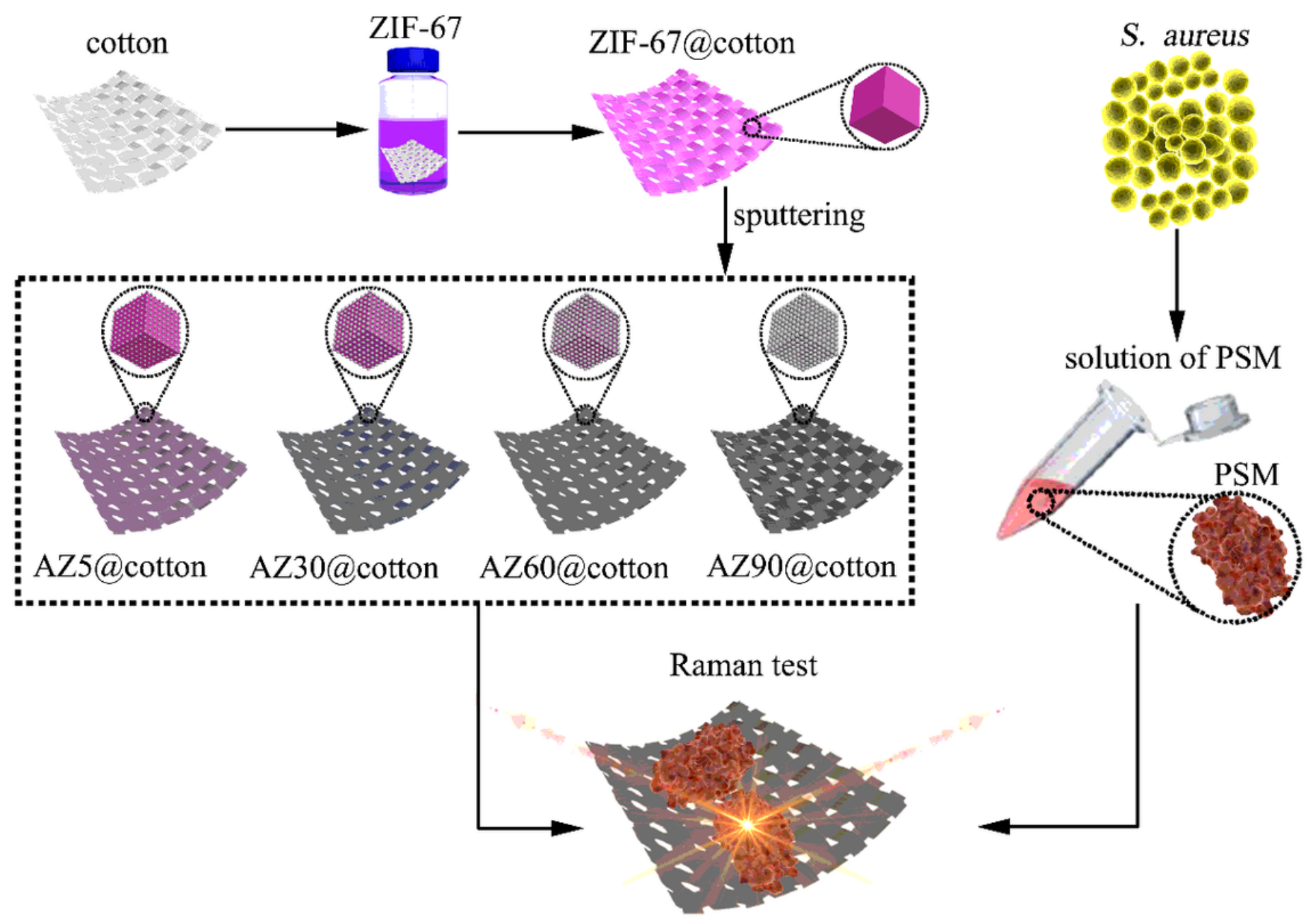

Figure 1

Schematic of fabrication and testing process 

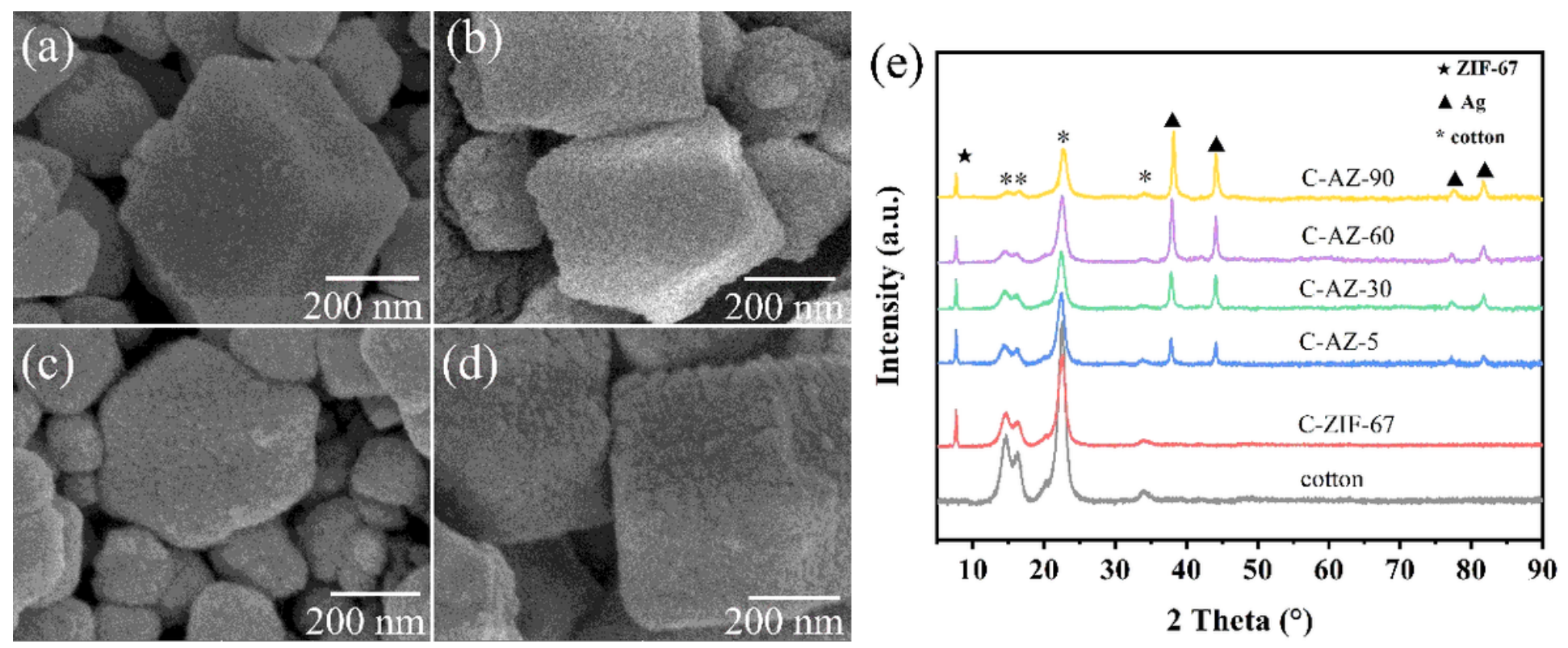

Figure 2

(a)-(d) SEM images of Ag nanoparticles sputtered onto C-ZIF-67 for 5 s, 30 s, 60 s and 90 s. (e) XRD patterns of cotton, C-ZIF-67 and C-Ag@ZIF-67
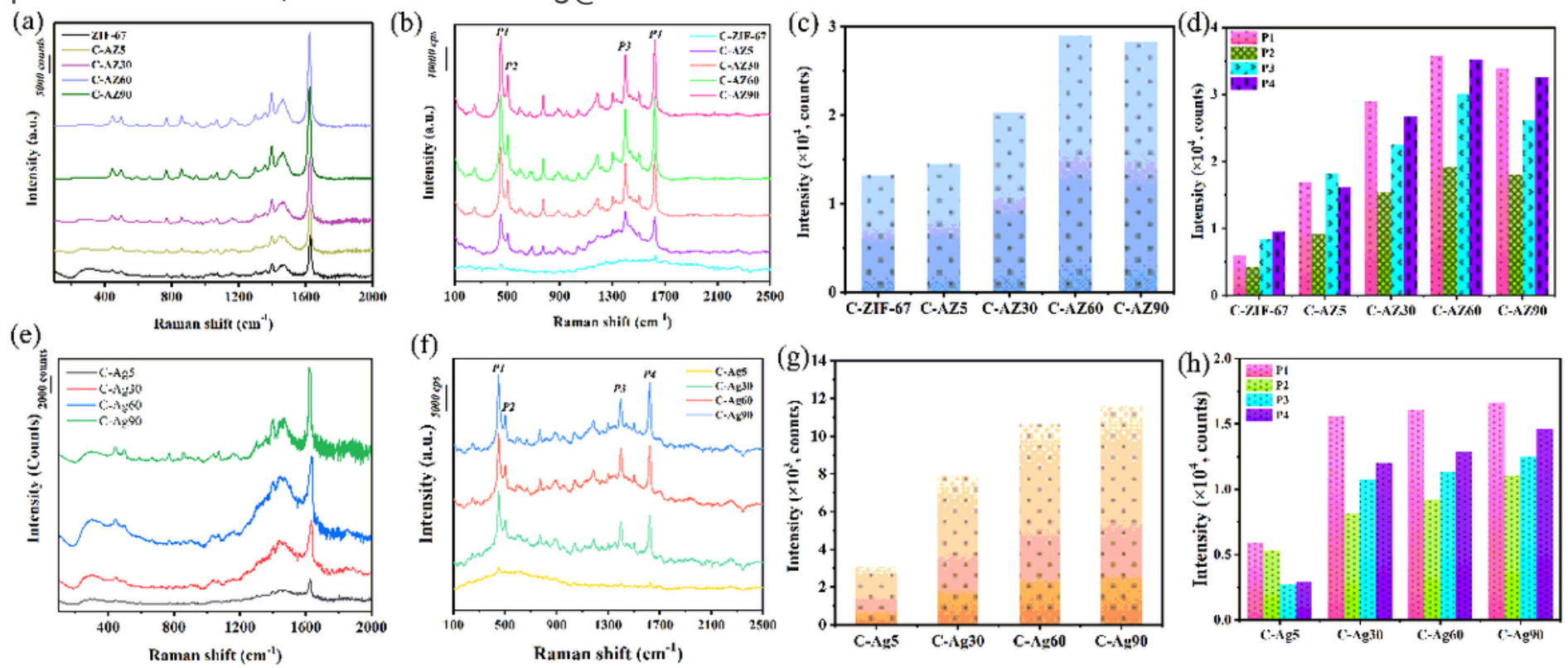

\section{Figure 3}

(a) and (c) Raman spectra and peak intensity of 1624 cm-1 of ZIF-67 and C-Ag@ZIF-67 at laser excitation of $532 \mathrm{~nm}$; (b) and (d) Raman spectra and peak intensity of 1624 cm-1 of ZIF-67 and C-Ag@ZIF-67 at laser excitation of $785 \mathrm{~nm}$; (e) and (f) Raman spectra and peak intensity of $1624 \mathrm{~cm}-1$ of C-Ag at laser excitation of $532 \mathrm{~nm}$; (f) and (h) Raman spectra and peak intensity of $1624 \mathrm{~cm}-1$ of C-Ag at laser excitation of $785 \mathrm{~nm}$ (all signals obtained from MB) 


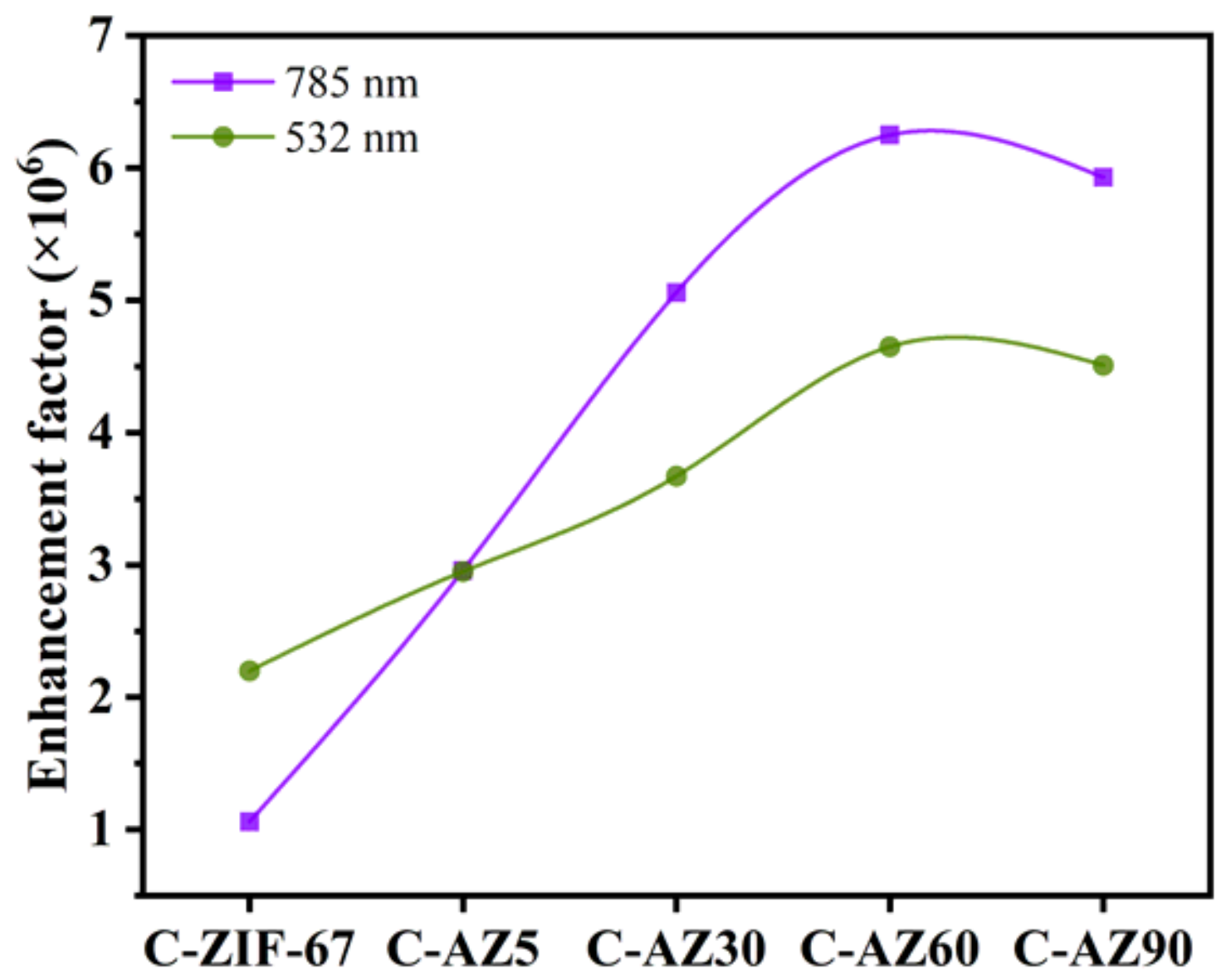

Figure 4

Enhancement factors of C-ZIF-67 and C-Ag@ZIF-67 

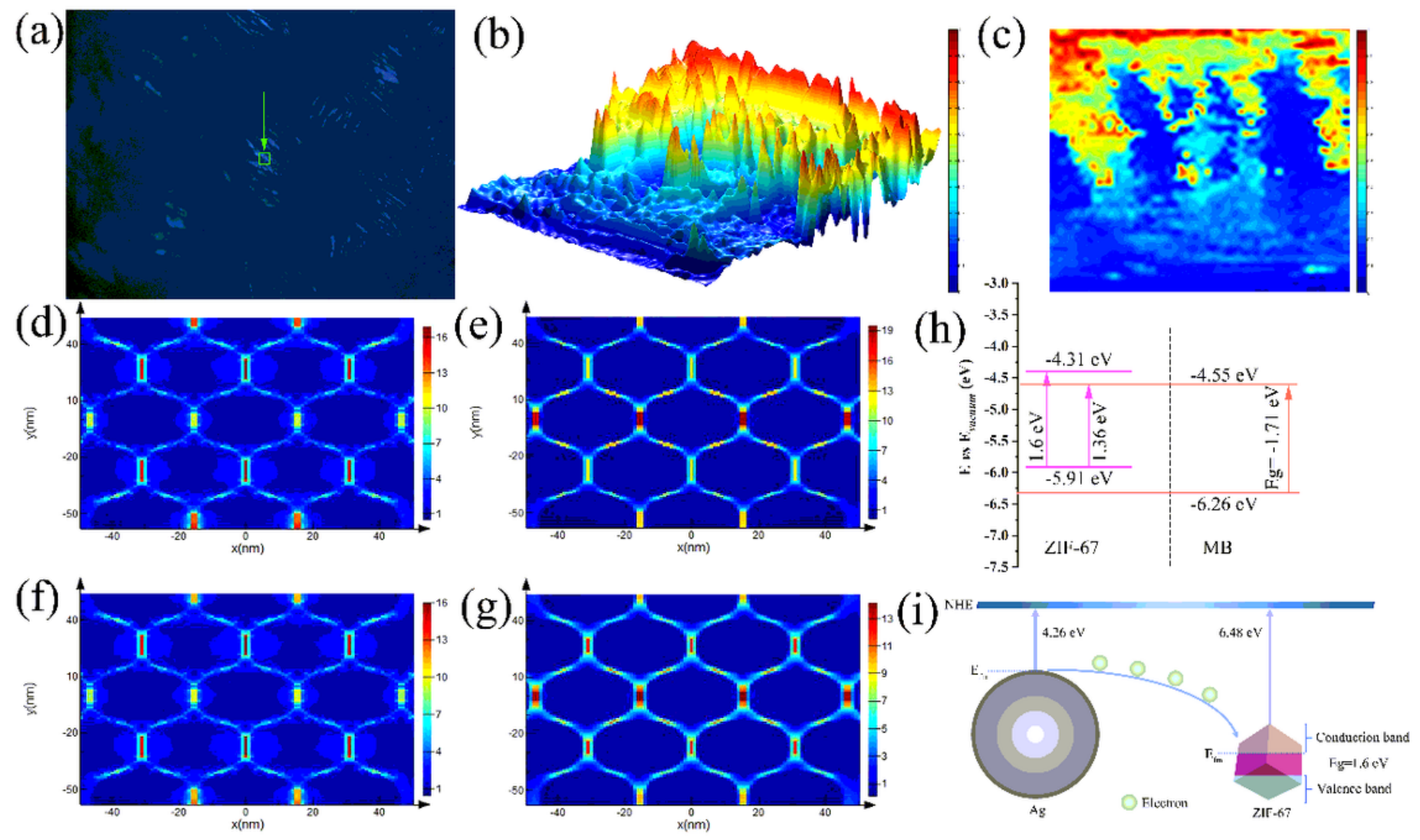

Figure 5

(a) Optical image of selected area for Raman mapping; (b) 3D Raman mapping; (c) 2D Raman mapping; (d) FDTD simulation of C-AZ60 at laser excitation of $532 \mathrm{~nm}$; (e) FDTD simulation of C-AZ60 at laser excitation of $785 \mathrm{~nm}$; (f) FDTD simulation of C-Ag60 at laser excitation of $532 \mathrm{~nm}$; (g) FDTD simulation of C-Ag60 at laser excitation of $785 \mathrm{~nm}$; (h) schematic of energy band gap matching between ZIF-67 and probe molecules for enhancement effect by charge transfer resonance; and (i) schematic of electron transfer between Ag and ZIF-67 

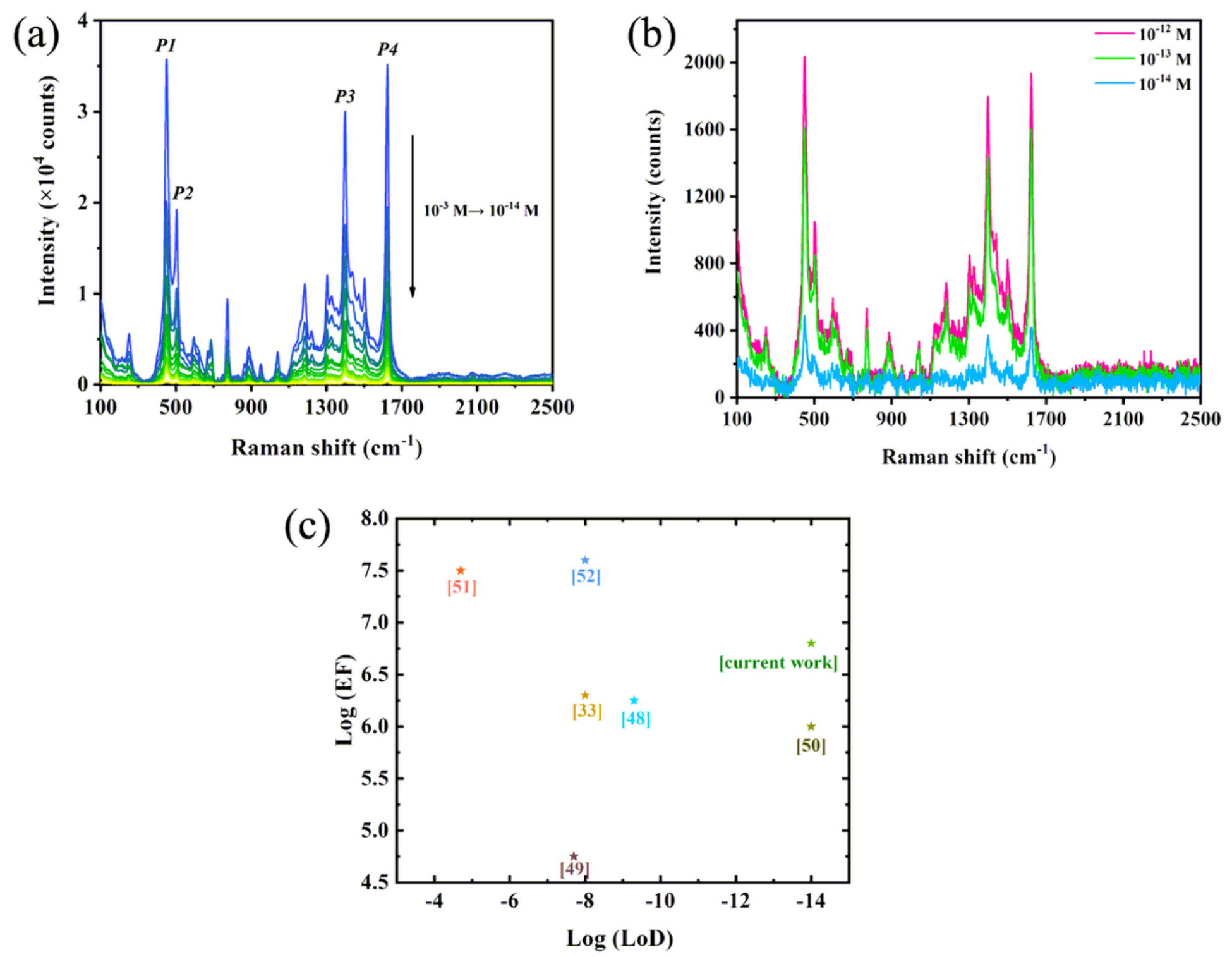

Figure 6

(a) and (b) Limitation of detection; (c) comparison of EFs and LoD: this study vs. other studies 

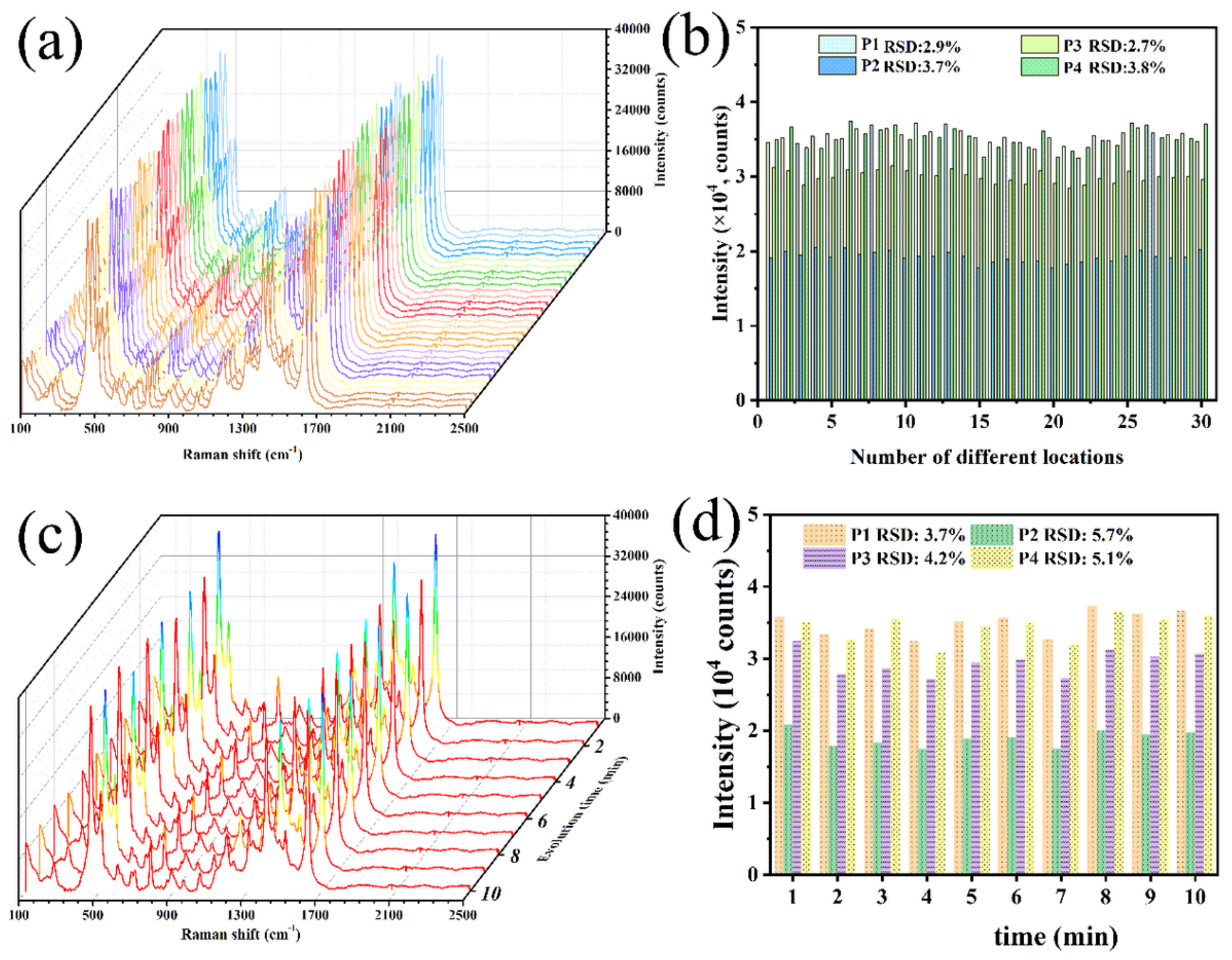

Figure 7

Plots of (a) and (b) signal reproducibility; and (c) and (d) signal stability 
(a)

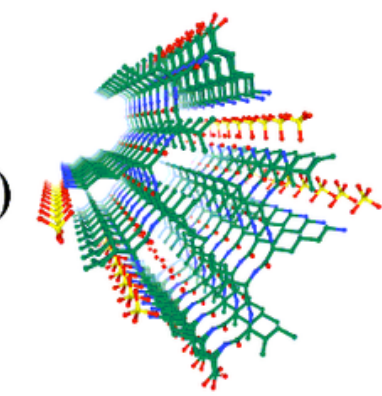

Peptide Sequence:

MGIIAGIIKVIKSLIEQFTGK

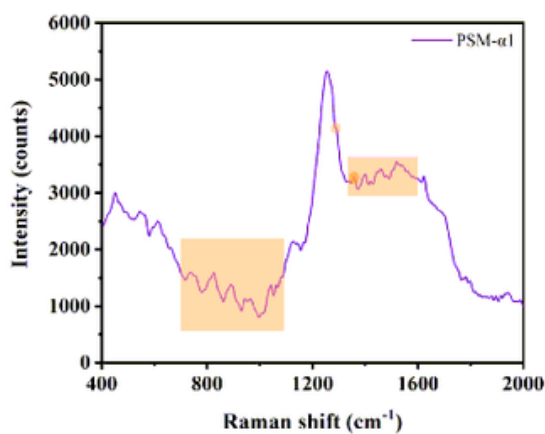

\section{PSM- $\alpha 1$}

(b)

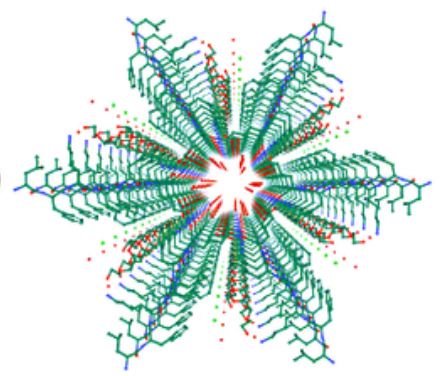

Peptide Sequence:

\section{MEFVAKLFKFFKDLLGKFLGNN}

PSM- $\alpha 3$

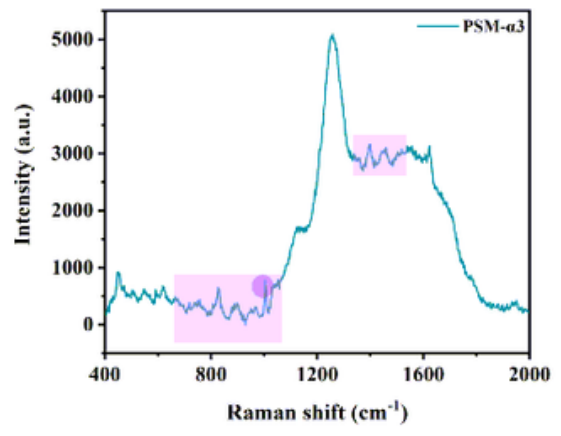

(c)

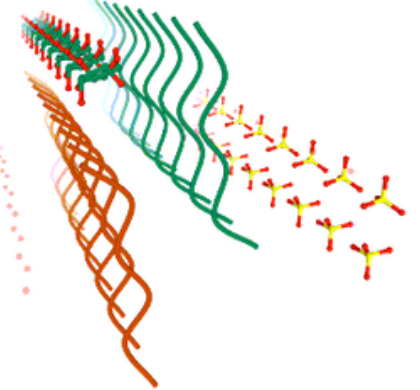

PSM- $\alpha 4$
Peptide Sequence:

MAIVGTIIKIIKAIIDIFAK

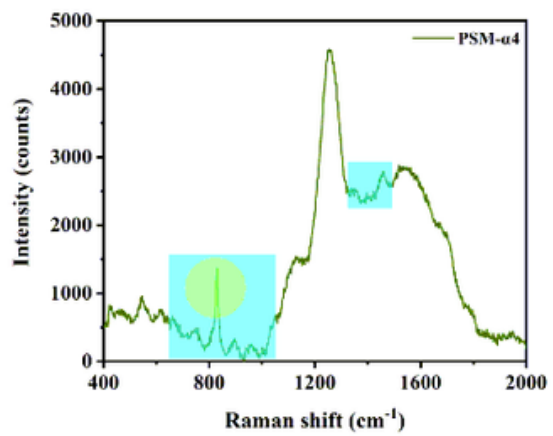

(d)

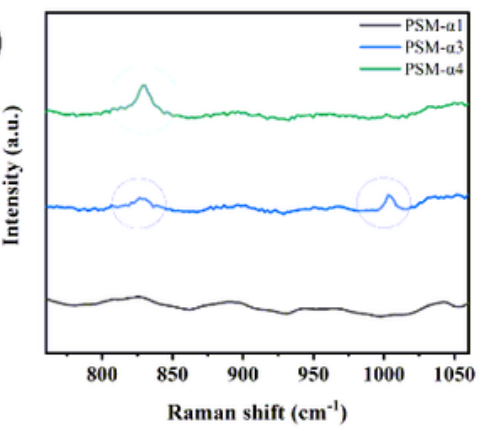

(e)

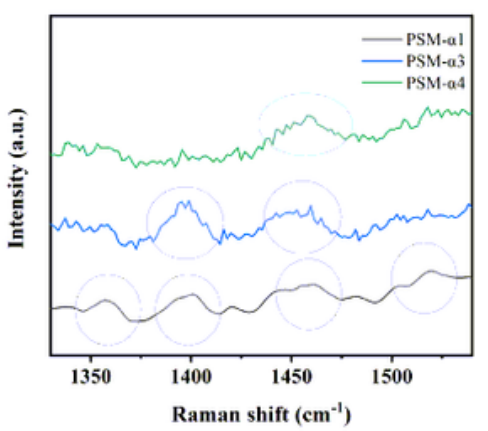

Figure 8

Structure, peptide sequence and Raman spectrum of (a) PSM a1, (b) a3, (c) a4, (d) local Raman spectra ranged from $760 \mathrm{~cm}-1 \sim 1060 \mathrm{~cm}-1$, and (e) local Raman spectra ranged from $1330 \mathrm{~cm}-1 \sim 1540 \mathrm{~cm}-1$

\section{Supplementary Files}


This is a list of supplementary files associated with this preprint. Click to download.

- Onlinefloatimage1.png

- supportinginfrmation.docx 